\title{
CONSISTENCY OF LINEAR INEQUALITIES OVER SETS
}

\author{
ABRAHAM BERMAN
}

\begin{abstract}
Necessary and sufficient conditions for the equivalence of the statements: (I) The system $b-A x \in T, x \in S$, is consistent. (II) $y \in T^{*}, A^{H} y \in S^{*} \Rightarrow \operatorname{Re}(b, y) \geqq 0$, are given in terms of the sets $S$ and $T$ and the matrix $A$. Sufficient conditions for this equivalence are obtained in the case where $S$ and $T$ are closed convex cones.
\end{abstract}

Introduction. A main tool in the theory of symmetric linear programming (e.g. [3]) is the following theorem:

Let $S \subset C^{n}$ and $T \subset C^{m}$ be polyhedral cones and let $A \in C^{m \times n}$. Then

(I) The system $b-A x \in T, x \in S$, is consistent if and only if

(II) $y \in T^{*}, A^{H} y \in S^{*} \Rightarrow \operatorname{Re}(b, y) \geqq 0$.

This equivalence does not hold for general cones, that is, there are nonpolyhedral cones for which (I) and (II) are not equivalent. On the other hand, there are sets $S$ and $T$ which are not cones such that for a suitable $A$, (I) and (II) are equivalent.

The main purpose of this paper is to characterize those triplets $(S, T, A)$ for which (I) and (II) are equivalent for every $b \in C^{n}$. This is done in $\S 2$ which follows a section of notation and preliminaries. The case where $S$ and $T$ are closed convex cones is described in the third section where sufficient conditions, including results of Ben-Israel [1], Fan [4] and Sposito-David [8], for the equivalence of -(I) and (II) are derived as corollaries.

1. Notation and preliminaries. $C^{n}$ denotes the $n$-dimensional complex vector space. $R^{n}$ denotes the $n$-dimensional real vector space. $R_{+}^{n}$ is the nonnegative orthant in $R^{n}$. $C^{m \times n}$ denotes the space of $m \times n$ complex matrices.

For $x, y \in C^{n}: \operatorname{Re} x$ is the real part of $x$,

$$
(x, y)=(\overline{y, x}) \text { is the inner product of } x \text { and } y .
$$

Presented to the Society, July 8, 1971; received by the editors July 28, 1971. AMS 1969 subject classifications. Primary 1550, 5230; Secondary 5210, 9050.

Key words and phrases. Affine set, closed convex cone, consistency, linear inequality, linear transformation, pointed cone, polar, polyhedral cone, relative interior. 
For $S, T \subset C^{n}: S \times T$ is the cartesian product.

$$
I_{n} \text { is the identity matrix of order } n \text {. }
$$

For $A \in C^{m \times n}: A^{H}$ is the conjugate transpose of $A, R(A)$ is the range of $A, N(A)$ is the null space of $A,[A, I]=\left[A, I_{m}\right], N[A, I]=\left\{[x, y] \in C^{n} \times C^{m}\right.$, $y=-A x\}$.

A nonempty set $S \subset C^{n}$ is

(i) convex if $0 \leqq \lambda \leqq 1 \Rightarrow \lambda S+(1-\lambda) S \subset S$,

(ii) a cone if $0 \leqq \lambda \Rightarrow \lambda S \subset S$,

(iii) a convex cone if it satisfies (i) and (ii),

(iv) a pointed cone if it satisfies (i) and $S \cap(-S)=0$,

(v) a polyhedral cone if $S=B R_{+}^{k}$, for some $B \in C^{n \times k}$.

A polyhedral cone is a closed convex cone. The polar, $S^{*}$, of $S$ is given by

$$
S^{*}=\left\{y \in C^{n} \mid x \in S \Rightarrow \operatorname{Re}(x, y) \geqq 0\right\} .
$$

If $S \subset T$, then $S^{*} \supset T^{*}$. The polar of a subspace is its orthogonal complement. In particular $(N(A))^{*}=R\left(A^{H}\right) . S^{*}$ is a closed convex cone. $S^{* *}=\left(S^{*}\right)^{*}$ is the smallest closed convex cone which contains $S$. Thus, $S^{* *}=S$ if and only if $S$ is a closed convex cone, and $S^{*}=S^{* * *}$.

The interior of $S^{*}$, int $S^{*}$, is given algebraically by

$$
\text { int } S^{*}=\left\{y \in S^{*} \mid 0 \neq x \in S \Rightarrow \operatorname{Re}(x, y)>0\right\} \text {. }
$$

For a cone $S$, int $S^{*} \neq \varnothing$ if and only if $S$ is pointed.

A set $S \subset C^{n}$ is affine if $(1-\lambda) S+\lambda S \subset S$ for all real $\lambda$. Every affine set is a translation of a subspace. The smallest affine set containing $S$, denoted by aff $S$, is called the affine hull of $S$. When $S$ is a convex cone, aff $S$ is a subspace.

The relative interior of a convex set $S$, denoted by ri $S$, is the interior which results when $S$ is regarded as a subset of aff $S$. When $S$ is a pointed cone, ri $S^{*}=$ int $S^{*}$. For more on cones, affine sets and relative interiors the reader is referred to [7], especially $\S \S 1$ and 6.

Let $S$ be a closed convex cone in $C^{n}, A \in C^{m \times n}, S$ the closed convex cone $S$ when regarded as a subset of aff $S$, and $S^{*}$ the polar cone of $S$ (in aff $S$ ). Decompose $C^{n}$ to aff $S \times(\text { aff } S)^{*}$ and define $\bar{A}$, a linear transformation from aff $S$ to $C^{m}$, by $\bar{A} x=A[x, \overline{0}]$ where $\overline{0}$ is the origin of (aff $S)^{*}$. Then ri $S=$ int $S, S^{*}=(\bar{S})^{*} \times(\text { aff } S)^{*}$ and $\bar{A}^{H} z$ is the perpendicular projection of $A^{H} z$ on aff $S$.

2. Consistency over sets. Triplets $S, T, A$ for which (I) and (II) are equivalent are characterized in the following theorem. 
THEOREM 1. Let $A \in C^{m \times n}, S \subset C^{m}$ and $T \subset C^{m}$. Then the following are equivalent:

(a) (I) and (II) are equivalent for every $b \in C^{n}$.

(b) $A S+T$ is a closed convex cone and $A S \cup T \subset A S+T$.

(c) $N(A, I)+S \times T$ is a closed convex cone and $A S \cup T \subset A S+T$.

Proof. First notice that

$$
A^{H} y \in S^{*} \Leftrightarrow y \in(A S)^{*}
$$

since both sides of (1) are equivalent to $x \in S \Rightarrow \operatorname{Re}(A x, y) \geqq 0$. From (1) it follows that (II) $\Leftrightarrow\left[y \in(A S)^{*} \cap T^{*} \Rightarrow \operatorname{Re}(b, y) \geqq 0\right] \Leftrightarrow b \in\left((A S)^{*} \cap T^{*}\right)^{*}$, while (I) $\Leftrightarrow b \in A S+T$. Thus (a) may be rewritten as

(a') $A S+T=\left((A S)^{*} \cap T^{*}\right)^{*}$.

We shall show that $\left(\mathrm{a}^{\prime}\right) \Leftrightarrow(\mathrm{b}) \Leftrightarrow(\mathrm{c})$.

$\left(\mathrm{a}^{\prime}\right) \Rightarrow(\mathrm{b}) . A S+T$ is a closed convex cone since it is a polar of a set. Taking polars of the two sides of $\left(\mathrm{a}^{\prime}\right)$ one gets

$$
(A S+T)^{*}=\left((A S)^{*} \cap T^{*}\right)^{* *}=(A S)^{*} \cap T^{*},
$$

since the intersection of two closed convex cones is a closed convex cone. Thus $(A S+T)^{*} \subset(A S)^{*}$ and $(A S+T)^{*} \subset T^{*}$, implying $A S \subset A S^{* *} \subset$ $(A S+T)^{* *}=A S+T$ and $T \subset T^{* *} \subset(A S+T)^{* *}=A S+T$ so that $A S \cup T \subset$ $A S+T$.

(b) $\Rightarrow\left(\mathrm{a}^{\prime}\right)$. It is always true that $(A S)^{*} \cap T^{*} \subset(A S+T)^{*}$. Also $A S \cup T \subset$ $A S+T \Rightarrow(A S+T)^{*} \subset(A S)^{*} \cap T^{*}$. Thus $(\mathrm{b}) \Rightarrow(A S)^{*} \cap T^{*}=(A S+T)^{*}$ and $\left((A S)^{*} \cap T^{*}\right)^{*}=(A S+T)^{* *}=A S+T$, since $A S+T$ is a closed convex cone.

(b) $\Leftrightarrow(\mathrm{c})$. Since the last parts of the conditions are identical, one has to show that $A S+T$ is a closed convex cone (in $C^{m}$ ) if and only if $N[A, I]+$ $S \times T$ is a closed convex cone (in $C^{m \times n}$ ).

Since $A S+T=[A, I](S \times T)$ it suffices to show that $N(A)+S$ is a closed convex cone if and only if $A S$ is one. This is an unpublished result of Robert A. Abrams. The proof here seems to be new. First, notice that $N(A)+S$ is the (unique) largest set whose image under $A$ is $A S$, that is

$$
A x \in A S \Leftrightarrow x \in N(A)+S .
$$

We now show that

$$
A x \in(A S)^{* *} \Leftrightarrow x \in(N(A)+S)^{* *} .
$$

To do this we notice that

$$
A x \in(A S)^{* *} \Leftrightarrow x \in\left(A^{H}(A S)^{*}\right)^{*} \Leftrightarrow x \in\left(S^{*} \cap R\left(A^{H}\right)\right)^{*},
$$


the two equivalences following from (1). Thus in order to prove (3) it suffices to show that $(N(A)+S)^{*}=R\left(A^{H}\right) \cap S^{*}$. It is clear that $R\left(A^{H}\right) \cap$ $S^{*} \subset(N(A)+S)^{*}$. To show the inclusion in the other direction let $y \in$ $(N(A)+S)^{*}$. Then $z \in N(A), \omega \in S, \lambda \in R \Rightarrow \operatorname{Re}(\lambda z+\omega, y) \geqq 0$.

As $\lambda$ can take any sign, $\operatorname{Re}(\lambda z+\omega, y)=\lambda \operatorname{Re}(z, y)+\operatorname{Re}(\omega, y) \geqq 0$ can only hold if $\operatorname{Re}(z, y)=0$. Thus $y \in(N(A))^{*}=R\left(A^{H}\right)$.

Also $0 \in N(A) \Rightarrow S \subset N(A)+S \Rightarrow(N(A)+S)^{*} \subset S^{*}$, which completes the proof of (3). Now the equivalence of (b) and (c) follows from (2), (3) and the fact that $A S$ and $(A S)^{* *}$ are contained in $R(A)$.

3. Consistency over cones. It is clear that $S$ and $T$ which satisfy the conditions of Theorem 1 do not have to be cones. However in most cases of interest in the system of (I), $S$ and $T$ are closed convex cones and this is what we assume in this section.

Theorem 2. Let $A \in C^{m \times n}$ and let $S \subset C^{n}, T \subset C^{m}$ be closed convex cones. Then any of the following conditions is sufficient for any of the conditions of Theorem 1.

(a) $N(A, I)+S \times T$ (or $A S+T)$ is closed.

(b) $N(A, I) \cap S \times T$ is a subspace.

(c) $S$ is pointed and the system $y \in T^{*}, A^{H} y \in$ int $S^{*}$, is consistent.

(d) $S$ and $T$ are pointed and the system $y \in \operatorname{int} T^{*}, A^{H} y \in$ int $S^{*}$, is consistent.

(e) $S$ and $T$ are polyhedral cones.

Proof. The condition that $A S \cup T \subset A S+T$ is satisfied since $S$ and $T$ are cones and thus $0 \in A S \cap T$. Condition (a) is stated for completeness. We show $(\mathrm{d}) \Rightarrow(\mathrm{c}) \Rightarrow(\mathrm{b}) \Rightarrow(\mathrm{a}) \Leftarrow(\mathrm{e})$.

(d) $\Rightarrow$ (c). Since $A^{H}$ is continuous, (c) is equivalent to

(c') $S$ is pointed and the system $y \in$ ri $T^{*}, A^{H} y \in$ int $S^{*}$, is consistent. If now $T$ is pointed, then (d) and (c) are the same.

(c) $\Rightarrow$ (b). Regarding $T^{*}$ as a subset of aff $T^{*},\left(c^{\prime}\right)$ becomes

(c) The system $y \in$ int $\bar{T}^{*}, \bar{A}^{H} y \in$ int $S^{*}$, is consistent.

By Corollary 1.4 of [2], this is equivalent to

$$
-\left(\bar{A}^{H}\right)^{H} z \in\left(\bar{T}^{*}\right)^{*}, \quad z \in S \Rightarrow z=0,
$$

which may be rewritten as: $\left(-\left(\bar{A}^{H}\right)^{H} z, t\right) \in\left(\bar{T}^{*}\right)^{*} \times\left(\right.$ aff $\left.\bar{T}^{*}\right), z \in S \Rightarrow z=0$, or $-A z \in T, z \in S \Rightarrow z=0$. Thus, $N[A, I] \cap S \times T=0$ which implies (b).

(b) $\Rightarrow($ a). Follows from Lemma 1.2 of [2] by replacing $A$ by $[A, I]$ and $S$ by $S \times T$.

$(\mathrm{e}) \Rightarrow(\mathrm{a})$. The image, by a linear transformation, of a polyhedral cone is a polyhedral cone. The sum of two polyhedral cones is a polyhedral cone. So $A S+T$ is a polyhedral cone and thus a closed convex cone.

The paper is concluded with two remarks on Theorem 2. 
1. Conditions (a) and (e) of Theorem 2, were proved, in the case where $T$ is the origin, by Ben-Israel [1, Theorems 2.4 and 3.5]. Special choices of $S$ include the Lemma of Farkas [5] (where $S=R_{+}^{n}$ ) and a theorem of Levinson [6]. Condition (c) of Theorem 2 is due to Sposito-David [8]. Condition (d) is a finite version of Theorem 5 of Fan [4].

2. The conditions in Theorem 2 are not equivalent. To show this let $T=C^{m}$ or $A=0$. In both cases (c) cannot hold and (a) holds, while (b) is true if and only if $S$ is a subspace.

The author wishes to thank the referee for his helpful remarks.

\section{REFERENCES}

1. A. Ben-Israel, Linear equations and inequalities on finite dimensional, real or complex, vector spaces: A unified theory, J. Math. Anal. Appl. 27 (1969), 367-389. MR 39 \#4192.

2. A. Berman and A. Ben-Israel, More on linear inequalities with applications to matrix theory, J. Math. Anal. Appl. 33 (1971), 482-496.

3. - Linear inequalities, mathematical programming and matrix theory, Math. Prog. 1 (1971), 291-300.

4. K. Fan, A generalization of the Alaoglu-Bourbaki theorem and applications, Math. Z. 88 (1965), 48-60. MR 31 \#2584.

5. J. Farkas, Über Theorie der einfachen Ungleichungen, J. Reine Angew. Math. 124 (1902), 1-24.

6. N. Levinson, Linear programming in complex space, J. Math. Anal. Appl. 14 (1966), 44-62. MR 37 \#1162.

7. R. T. Rockafellar, Convex analysis, Princeton Math. Series, no. 28, Princeton Univ. Press, Princeton, N.J., 1970. MR 43 \#445.

8. V. A. Sposito and H. T. David, A note on Farkas lemmas over cone domains, Iowa State University Report.

Centre de Recherches Mathématiques, Université de Montréal, Montréal, QUÉBEC, CANADA

Current address: Department of Mathematics, Technion-Israel Institute of Technology, Haifa, Israel 